\title{
INJECTING WORD INFORMATION WITH MULTI-LEVEL WORD ADAPTER FOR CHINESE SPOKEN LANGUAGE UNDERSTANDING
}

\author{
Dechuan Teng ${ }^{\star} \quad$ Libo Qin ${ }^{\star} \quad$ Wanxiang Che $e^{\dagger} \quad$ Sendong Zhao Ting Liu \\ Research Center for Social Computing and Information Retrieval, Harbin Institute of Technology, China
}

\begin{abstract}
In this paper, we improve Chinese spoken language understanding (SLU) by injecting word information. Previous studies on Chinese SLU do not consider the word information, failing to detect word boundaries that are beneficial for intent detection and slot filling. To address this issue, we propose a multi-level word adapter to inject word information for Chinese SLU, which consists of (1) sentence-level word adapter, which directly fuses the sentence representations of the word information and character information to perform intent detection and (2) character-level word adapter, which is applied at each character for selectively controlling weights on word information as well as character information. Experimental results on two Chinese SLU datasets show that our model can capture useful word information and achieve state-of-the-art performance.
\end{abstract}

Index Terms - Chinese Spoken Language Understanding, Intent Detection, Slot Filling, Word Adapter

\section{INTRODUCTION}

Spoken language understanding plays an important role in task-oriented dialog systems, which mainly consists of two subtasks including slot filling and intent detection [1]. Take the sentence "use netflix to play music" as an example, intent detection aims to classify the intent label (i.e., P layMus ic) for the whole sentence, while slot filling aims to assign different slot labels (i.e., O, B-service, O, O, O) for each token in this sentence.

Compared with SLU in English, Chinese SLU faces a unique challenge since it usually needs word segmentation. Nevertheless, the imperfect segmentation performed by the CWS (Chinese Word Segmentation) system will misidentify slot boundaries and predict wrong slot categories, therefore suffers from the error propagation. To address this issue, Liu et al. [2] proposed a character-based method to perform Chinese SLU in a joint model at the character level, achieving state-of-the-art performance.

However, the main drawback of the character-based SLU model is that explicit word sequence information is not fully

\footnotetext{
* Equal contributions

$\dagger$ Corresponding author.
}

exploited, which might be useful for understanding Chinese texts. Take the Chinese utterance “周冬雨 (Dongyu Zhou) / 有 (has) / 哪些 (which) / 电影 (movies)” as an example, where the utterance is split into a sequence of words by “"”. The character-based model is easily confused and predicts “周 (week)” as Datetime_date and treats “冬雨 (winter rain)" as Datetime_time wrongly. In contrast, with the help of words such as “周冬雨 (Dongyu Zhou)", a model can easily detect the correct slot label Artist. Thus, it's important to incorporate words into Chinese SLU tasks. Unfortunately, there has been a limited amount of research on how to introduce word information to enhance Chinese SLU effectively.

To bridge the gap, we propose a simple but effective method for injecting word information into Chinese SLU. Since SLU is unique in jointly modeling two correlated tasks, exploiting their interaction can be useful for modeling fine-grained word information transfer between two tasks. To this end, we design a multi-level word adapter to perform slot filling and intent detection in a joint model: (1) sentence-level word adapter directly fuses the word-aware and character-aware sentence representations for identifying intents; (2) character-level word adapter adaptively determines the weights between character features and word features for assigning slot labels for each character, so that finegrained combinations of word knowledge can be achieved. In addition, the proposed word adapter is applied as a plugin for the output layer, with no need to change other components in the original character-based model, achieving more flexibility.

The experimental results show the effectiveness of our framework by outperforming the SOTA methods by a large margin. All datasets and codes will be publicly available at https: / / github.com/AaronTengDeChuan/ MLWA-Chinese-SLU.

\section{APPROACH}

\subsection{Overview}

We build our model based on a vanilla character-based model, as shown in Figure 1(a). To explicitly incorporate word information, as shown in 1 (b), we propose a multi-level word 


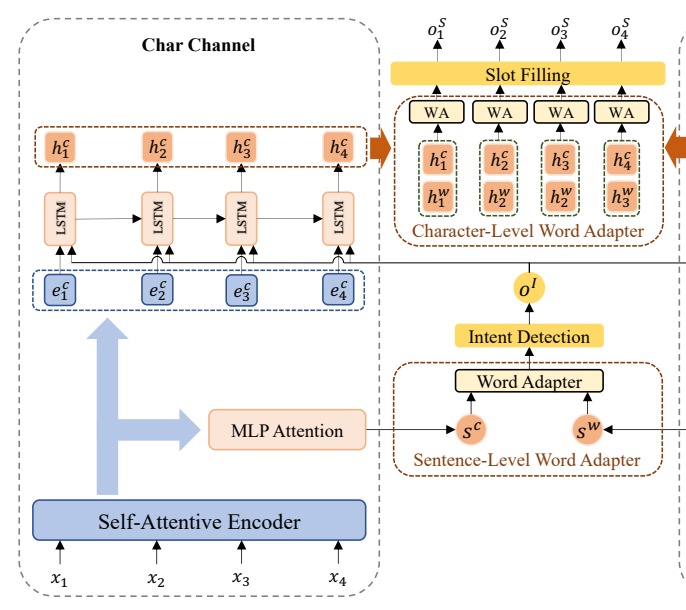

(a)

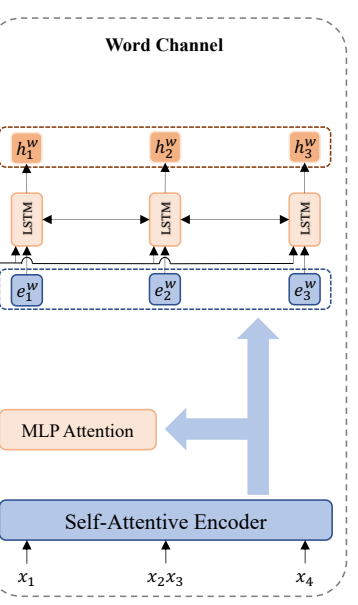

(b)

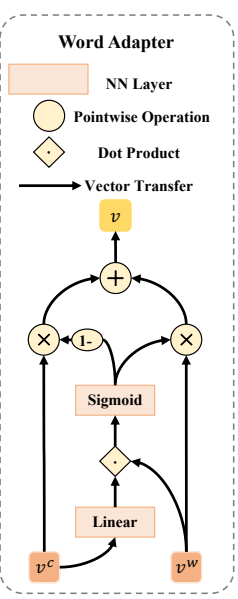

(c)

Fig. 1. Illustration of our proposed Multi-Level Word Adapter for Chinese SLU, which consists of a sentence-level word adapter for intent detection and a character-level word adapter for slot filling. The internal structure of a word adapter is shown in (c).

adapter to capture sentence-level and character-level word information for intent detection and slot filling.

\subsection{Vanilla Character-based Model}

The vanilla character-based model, as shown in Figure 1 a), consists of a shared self-attentive encoder, an intent detection decoder, and a slot filling decoder.

Char-Channel Encoder Following Qin et al. [3], we adopt a shared self-attentive encoder to obtain the character encoding representations. It mainly consists of a self-attention mechanism to extract the contextual information and a BiLSTM [4] to capture sequential information within characters.

Formally, the BiLSTM takes the input utterance as input, conduct recurrent steps forwardly and backwardly, and obtain context-sensitive sequential features. The idea of the selfattention module from Vaswani et al. [5] is to capture contextualized representation for each token in a sequence that has shown effective in SLU tasks [3]. Finally, given a Chinese utterance $\mathbf{c}=\left\{c_{1}, c_{2}, \cdots, c_{N}\right\}$ with $N$ characters, we concatenate the output of BiLSTM and self-attention over character sequence $\mathbf{c}$ to obtain the final encoding representations $\mathbf{E}^{c}=$ $\left\{\mathbf{e}_{1}^{c}, \mathbf{e}_{2}^{c}, \cdots, \mathbf{e}_{N}^{c}\right\}$.

Intent Detection We first apply a MLP attention module [6, 7] to obtain the whole utterance representation $\mathbf{s}^{c}$ by calculating the weighted sum of all hidden states $\mathbf{e}_{t}^{c}$ in $\mathbf{E}^{c}$. Then, we take the overall vector $\mathbf{s}^{c}$ to perform intent detection as follows:

$$
\begin{aligned}
P\left(\widetilde{y}=j \mid \mathbf{s}^{c}\right) & =\operatorname{softmax}\left(\mathbf{W}^{I} \mathbf{s}^{c}+\mathbf{b}^{I}\right) \\
\mathbf{o}^{I} & =\underset{\widetilde{y} \in S^{\text {int }}}{\arg \max } P\left(\widetilde{y} \mid \mathbf{s}^{c}\right)
\end{aligned}
$$

where $S^{\text {int }}$ is the intent label set, and $\mathbf{W}^{I}, \mathbf{b}^{I}$ are trainable parameters.
Slot Filling We use a unidirectional LSTM as the slot filling decoder and follow prior works [3] to leverage the intent information to guide the slot prediction. Previous studies have demonstrated the superiority of exploiting explicit information from closely correlated task [3, 8]. At each decoding step $t$, the decoder hidden state $\mathbf{h}_{t}^{c, S}$ is calculated as follows:

$$
\mathbf{h}_{t}^{c, S}=\operatorname{LSTM}\left(\mathbf{e}_{t}^{c} \oplus \phi^{\text {int }}\left(\mathbf{o}^{I}\right) \oplus \mathbf{y}_{t-1}^{S}, \mathbf{h}_{t-1}^{c, S}\right)
$$

where $\phi^{\text {int }}(\cdot)$ represents the embedding matrix of intents, and $\mathbf{y}_{t-1}^{S}$ is the embedding of the emitted slot label from previous decoding step. Then, the hidden state $\mathbf{h}_{t}^{c, S}$ is utilized to perform slot filling:

$$
\begin{aligned}
P\left(\widetilde{y}=j \mid \mathbf{h}_{t}^{c, S}\right) & =\operatorname{softmax}\left(\mathbf{W}^{S} \mathbf{h}_{t}^{c, S}+\mathbf{b}^{S}\right) \\
\mathbf{o}_{t}^{S} & =\underset{\widetilde{y} \in S^{s l o t}}{\arg \max } P\left(\widetilde{y} \mid \mathbf{h}_{t}^{c, S}\right) \\
\mathbf{y}_{t}^{S} & =\phi^{\text {slot }}\left(\mathbf{o}_{t}^{S}\right)
\end{aligned}
$$

where $\mathbf{W}^{S}$ and $\mathbf{b}^{S}$ are trainable parameters, $S^{\text {slot }}$ is the slot label set, $\mathbf{o}_{t}^{S}$ is the slot label of $t$ th character $c_{t}$, and $\phi^{\text {slot }}(\cdot)$ represents the embedding matrix of slots.

\subsection{Multi-Level Word Adapter}

The multi-level word adapter is the core module for combine character and word features, which can be used as a plugin to the above character-based model. In particular, it consists of a word-channel encoder to obtain the word encoding information, a sentence-level word adapter to enhance intent detection at the sentence level, and a character-level word adapter to boost slot filling at the character level.

Word-Channel Encoder In our framework, the word-channel encoder is independent of the char-channel encoder, that is, 
we can freely decide how to utilize word information, without considering the interference between two encoders.

When using an external CWS system, we conduct word segmentation over the utterance $\mathbf{c}$ and obtain the word sequences $\mathbf{w}=\left\{w_{1}, w_{2}, \cdots, w_{M}\right\}(M \leq N)$. Same with char channel encoder, the word channel encoder generates the final word-channel representations $\mathbf{E}^{w}=\left\{\mathbf{e}_{1}^{w}, \mathbf{e}_{2}^{w}, \cdots, \mathbf{e}_{M}^{w}\right\}$.

Word Adapter The word adapter shown in Figure 11 (c) is a simple neural network layer for adaptively integrating character and word features. Given the input character vector $\mathbf{v}^{c} \in \mathbb{R}^{d}$ and word vector $\mathbf{v}^{w} \in \mathbb{R}^{d}$, we calculate the weight between two input vectors and then conduct a weighted sum of these two vectors:

$$
\begin{aligned}
\mathrm{WA}\left(\mathbf{v}^{c}, \mathbf{v}^{w}\right) & =(1-\lambda) \cdot \mathbf{v}^{c}+\lambda \cdot \mathbf{v}^{w} \\
\lambda & =\operatorname{sigmoid}\left(\mathbf{v}^{c \top} \mathbf{W}_{f} \mathbf{v}^{w}\right)
\end{aligned}
$$

where $\mathbf{W}_{f} \in \mathbb{R}^{d \times d}$ is trainable parameters and $\lambda$ can adaptively adjust the importance between character and word information.

Sentence-Level Word Adapter Given contextualized representations $\mathbf{E}^{c}$ and $\mathbf{E}^{w}$ over character and word sequences, we first obtain two summary vectors $\mathbf{s}^{c}$ and $\mathbf{s}^{w}$ using the MLP attention module. Then, a sentence-level word adapter calculate the fused summarized vector $\mathbf{v}^{I}=\mathrm{WA}\left(\mathbf{s}^{c}, \mathbf{s}^{w}\right)$ and then use it to predict the intent label $\mathbf{o}^{I}$ with the Equation (1).

$$
P\left(\widetilde{y}=j \mid \mathbf{v}^{I}\right)=\operatorname{softmax}\left(\mathbf{W}^{I} \mathbf{v}^{I}+\mathbf{b}^{I}\right)
$$

Character-Level Word Adapter For slot filling, we first adopt a bidirectional LSTM to strengthen slot-aware word representations. At each time step $i$, the hidden state $\mathbf{h}_{i}^{w, S}=$ $\operatorname{BiLSTM}\left(\mathbf{e}_{i}^{w} \oplus \phi^{\text {int }}\left(\mathbf{o}^{I}\right), \mathbf{h}_{i-1}^{w, S}\right)$ is derived from the corresponding word representation $\mathbf{e}_{i}^{w}$ and the embedding $\phi^{\text {int }}\left(\mathbf{o}^{I}\right)$ of the predicted intent $\mathbf{o}^{I}$.

Then, a character-level word adapter is adopted to determine different integration weights for different combinations of character and word features for each character 1

$$
\mathbf{v}_{t}^{S}=\mathrm{WA}\left(\mathbf{h}_{t}^{c, S}, \mathbf{h}_{f_{\text {align }}^{w, S}(t, \mathbf{w})}^{w}\right)
$$

Finally, we utilize the integration representation $\mathbf{v}_{t}^{S}$ that contains the word information and character information instead of $\mathbf{h}_{t}^{c, S}$ to perform slot filling with the Equation 3 :

$$
P\left(\widetilde{y}=j \mid \mathbf{v}_{t}^{S}\right)=\operatorname{softmax}\left(\mathbf{W}^{S} \mathbf{v}_{t}^{S}+\mathbf{b}^{S}\right)
$$

Joint Training Following Goo et al. [9], we adopt a joint training scheme for optimization, where the final joint objective function is computed as follows:

$$
\mathcal{L}=-\log P\left(\hat{y}^{I} \mid \mathbf{v}^{I}\right)-\sum_{i=1}^{N} \log P\left(\hat{y}_{i}^{S} \mid \mathbf{v}_{i}^{S}\right)
$$

\footnotetext{
${ }^{1}$ len $($ 周冬雨 $)=3, \mathbb{I}($ True $)=1$, and $\mathbb{I}($ False $)=0$. Given a word sequence $\mathbf{w}=\{$ “周冬雨”, “有”, “哪些”, “电影” $\}, f_{\text {align }}(t, \mathbf{w})$ gives the position index of the word corresponding to the $t$ th character in $\mathbf{w}$ (e.g., $\left.f_{\text {align }}(3, \mathbf{w})=1, f_{\text {align }}(4, \mathbf{w})=2, f_{\text {align }}(6, \mathbf{w})=3\right)$.
}

where $\hat{y}^{I}$ and $\hat{y}_{i}^{S}$ are golden intent and slot labels, respectively.

\section{EXPERIMENTS}

\subsection{Experimental Setup}

To verify the effectiveness of our proposed method, we conduct experiments on two Chinese SLU datasets, including CAIS [2] and ECDT-NLU ${ }^{2}$ CAIS dataset includes 7,995 training, 994 validation and 1024 test utterances. ECDT-NLU dataset consists of 2576 training and 1033 test utterances.

Following Goo et al. [9] and Qin et al. [3], we evaluate the Chinese SLU performance of slot filling with F1 score and the performance of intent prediction with accuracy, and sentence-level semantic frame parsing with overall accuracy. Overall accuracy denotes that the output for an utterance is considered as a correct prediction if and only if the intent and all slots exactly match its ground truth values.

We adopt the Chinese natural language processing system (Language Technology Platform, LTP [11]), to obtain the word segmentation 3 We use Adam optimizer [12] with the learning rate of 1e-3 to optimize all trainable parameters.

\subsection{Main Results}

Table 1 shows the main results of the proposed model and all compared baselines on the CAIS and ECDT-NLU datasets. From the results, we have the following observations: (1) Our model outperforms all baselines on all metrics by a large margin on two datasets and achieves state-of-the-art performance, which demonstrates the effectiveness of our proposed multi-level word adapters; (2) Our model achieves $0.97 \%$ improvement on Slot $\left(F_{1}\right)$ score, $0.79 \%$ improvement on Intent (Acc) on CAIS dataset, compared with SOTA model StackPropagation. On the ECDT-NLU dataset, we achieve $4.12 \%$ and $3.38 \%$ improvements on Slot $\left(F_{1}\right)$ score and Intent Accuracy. These improvements indicate that incorporating word information with proposed multi-level word adapters can benefit the Chinese intent detection and slot filling; (3) In particular, we obtain the obvious improvements on sentence-level semantic frame accuracy over CAIS $(+1.49 \%)$ and ECDT-NLU $(+2.52 \%)$ datasets. We achieve significant improvements due to 1) considering the correlation between two tasks and 2) the mutual enhancement with joint training.

\subsection{Analysis}

To verify the effectiveness of the proposed word adapters, we conduct the following ablation analysis.

Effect of Sentence-Level Word Adapter We only remove the sentence-level word adapter for intent detection. The w/o

2 http://conference.cipsc.org.cn/smp2019/ evaluation.html

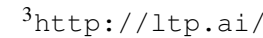




\begin{tabular}{l|ccc|ccc}
\hline \multirow{2}{*}{ Models } & \multicolumn{3}{|c|}{ CAIS } & \multicolumn{3}{c}{ ECDT-NLU } \\
\cline { 2 - 7 } & Slot $\left(F_{1}\right)$ & Intent $(A c c)$ & Overall $(A c c)$ & Slot $\left(F_{1}\right)$ & Intent $(A c c)$ & Overall $(A c c)$ \\
\hline Slot-Gated [9] & 81.13 & 94.37 & 80.83 & 46.96 & 72.41 & 26.72 \\
SF-ID Network [10] & 84.85 & 94.27 & 82.41 & 41.65 & 74.83 & 25.07 \\
CM-Net [2] & 86.16 & 94.56 & - & - & - & - \\
Stack-Propagation [3] & 87.64 & 94.37 & 84.68 & 46.98 & 78.03 & 31.56 \\
\hline Our Model & $\mathbf{8 8 . 6 1}$ & $\mathbf{9 5 . 1 6}$ & $\mathbf{8 6 . 1 7}$ & $\mathbf{5 1 . 1 0}$ & $\mathbf{8 1 . 4 1}$ & $\mathbf{3 4 . 0 8}$ \\
\hline
\end{tabular}

Table 1. Main Results on CAIS and ECDT-NLU.

\begin{tabular}{l|ccc|ccc}
\hline \multirow{2}{*}{ Models } & \multicolumn{3}{|c|}{ CAIS } & \multicolumn{3}{c}{ ECDT-NLU } \\
\cline { 2 - 7 } & Slot $\left(F_{1}\right)$ & Intent $(A c c)$ & Overall $(A c c)$ & Slot $\left(F_{1}\right)$ & Intent $(A c c)$ & Overall $(A c c)$ \\
\hline w/o Multiple Levels & 82.91 & 95.06 & 80.43 & 50.71 & 77.64 & 32.62 \\
w/o Sentence-Level word adapter & 86.31 & 94.96 & 83.99 & 50.26 & 79.96 & 33.01 \\
w/o Character-Level word adapter & 88.28 & 94.66 & 85.18 & 48.96 & 81.22 & 32.14 \\
\hline Full Model & $\mathbf{8 8 . 6 1}$ & $\mathbf{9 5 . 1 6}$ & $\mathbf{8 6 . 1 7}$ & $\mathbf{5 1 . 1 0}$ & $\mathbf{8 1 . 4 1}$ & $\mathbf{3 4 . 0 8}$ \\
\hline
\end{tabular}

Table 2. Ablation study on CAIS and ECDT-NLU datasets.

Sentence-Level word adapter row in Table 2 witnesses an obvious decline for the intent accuracy on ECDT-NLU, which demonstrates that the proposed word adapter can extract word information to promote the Chinese intent detection.

Effect of Character-Level Word Adapter From the results in w/o Character-Level word adapter row, we can observe $2.14 \%$ and $0.33 \%$ drops on slot F1 score on ECDT-NLU and CAIS, respectively. The drops show that word information can help the model to detect the word boundary, and the proposed character-level word adapter can successfully extract word information to improve slot filling.

Effect of Multiple Levels To investigate the effectiveness of the proposed multi-level mechanism, we conduct an experiment to remove the character-level word adapter and provide the same word information for all slots, which is named as w/o multiple level. From the results shown in Table 2 , we can observe that the character-level word adapter bring the significant positive effects. The speculation that each token needs different word information at a fine-grained level can explain the effectiveness of incorporating token-level word information for character-level slot filling task.

\section{RELATED WORK}

Intent Detection and Slot Filling Different methods, including support vector machine (SVM), recurrent neural networks (RNN), and attention-based models [13, 14, 15], have been proposed to perform intent detection. For slot filling, conditional random fields (CRF) and RNN were first proposed, and recent approaches are based on self-attention mechanisms [16, 17].

Recent works adopted joint models to consider their close relationship between slot filling and intent detection [18, 19 , 20, 9, 21, 3]. However, the above works are restricted to the English language. In contrast, we consider injecting word information for Chinese SLU in a joint model.
Chinese Spoken Language Understanding To avoid the imperfect CWS and leverage the character features, Liu et al. [2] proposed a character-based joint model to perform Chinese SLU. Compared with the work, we propose a multilevel word adapter to incorporate word information at both sequence level and character level for intent detection and slot filling. Word information has shown effectiveness in various Chinese NLP tasks including Chinese word segmentation [22, 23] and Chinese text classification [24, 25]. These work can be regarded as applications of incorporating word information into subproblems in SLU. In contrast with their work, we exploit mutual benefits between intent detection and slot filling for fine-grained word knowledge transfer. To the best of our knowledge, we are the first to incorporate word information to enhance Chinese SLU in a joint model.

\section{CONCLUSION}

In this work, we proposed a simple but effective method named multi-level word adapter to inject word information for Chinese SLU tasks. In particular, we introduced a sentence-level word adapter to inject word information for intent detection and a character-level word adapter to incorporate word information for slot filling. To the best of our knowledge, we are the first to explore injecting word information into Chinese SLU. The experimental results on two Chinese SLU datasets showed that our model improves performance significantly and achieves the best results.

\section{ACKNOWLEDGEMENTS}

This work was supported by the National Key R\&D Program of China via grant 2020AAA0106501 and the National Natural Science Foundation of China (NSFC) via grant 61976072 and 61772153 . This work was also supported by the Zhejiang Lab's International Talent Fund for Young Professionals. 


\section{REFERENCES}

[1] Gokhan Tur and Renato De Mori, Spoken language understanding: Systems for extracting semantic information from speech, John Wiley \& Sons, 2011.

[2] Yijin Liu, Fandong Meng, Jinchao Zhang, Jie Zhou, Yufeng Chen, and Jinan $\mathrm{Xu}$, "Cm-net: A novel collaborative memory network for spoken language understanding," in Proc. EMNLP, 2019, pp. 1050-1059.

[3] Libo Qin, Wanxiang Che, Yangming Li, Haoyang Wen, and Ting Liu, "A stack-propagation framework with token-level intent detection for spoken language understanding," in Proc. EMNLP, 2019, pp. 2078-2087.

[4] Sepp Hochreiter and Jürgen Schmidhuber, "Long shortterm memory," Neural computation, vol. 9, no. 8, pp. 1735-1780, 1997.

[5] Ashish Vaswani, Noam Shazeer, Niki Parmar, Jakob Uszkoreit, Llion Jones, Aidan N Gomez, Łukasz Kaiser, and Illia Polosukhin, "Attention is all you need," in Proc. NIPS, 2017, pp. 5998-6008.

[6] Victor Zhong, Caiming Xiong, and Richard Socher, "Global-locally self-attentive encoder for dialogue state tracking," in Proc. ACL, 2018, pp. 1458-1467.

[7] Rui Zhang, Cicero dos Santos, Michihiro Yasunaga, Bing Xiang, and Dragomir Radev, "Neural coreference resolution with deep biaffine attention by joint mention detection and mention clustering," in Proc. ACL, 2018, pp. 102-107.

[8] Sendong Zhao, Ting Liu, Sicheng Zhao, and Fei Wang, "A neural multi-task learning framework to jointly model medical named entity recognition and normalization," in Proc. AAAI, 2019, vol. 33, pp. 817-824.

[9] Chih-Wen Goo, Guang Gao, Yun-Kai Hsu, Chih-Li Huo, Tsung-Chieh Chen, Keng-Wei Hsu, and Yun-Nung Chen, "Slot-gated modeling for joint slot filling and intent prediction," in Proc. NAACL, 2018, pp. 753-757.

[10] Haihong E, Peiqing Niu, Zhongfu Chen, and Meina Song, "A novel bi-directional interrelated model for joint intent detection and slot filling," in Proc. ACL, 2019.

[11] Wanxiang Che, Yunlong Feng, Libo Qin, and Ting Liu, "N-ltp: A open-source neural chinese language technology platform with pretrained models," arXiv preprint arXiv:2009.11616, 2020.

[12] Diederik P Kingma and Jimmy Ba, "Adam: A method for stochastic optimization," arXiv preprint arXiv:1412.6980, 2014.
[13] Patrick Haffner, Gokhan Tur, and Jerry H Wright, “Optimizing svms for complex call classification," in Proc. ICASSP. IEEE, 2003, vol. 1, pp. I-I.

[14] Ruhi Sarikaya, Geoffrey E Hinton, and Bhuvana Ramabhadran, "Deep belief nets for natural language callrouting," in Proc. ICASSP. IEEE, 2011, pp. 5680-5683.

[15] Congying Xia, Chenwei Zhang, Xiaohui Yan, Yi Chang, and S Yu Philip, "Zero-shot user intent detection via capsule neural networks," in Proc. EMNLP, 2018, pp. 3090-3099.

[16] Tao Shen, Tianyi Zhou, Guodong Long, Jing Jiang, Shirui Pan, and Chengqi Zhang, "Disan: Directional self-attention network for rnn/cnn-free language understanding," in Proc. AAAI, 2018.

[17] Zhixing Tan, Mingxuan Wang, Jun Xie, Yidong Chen, and Xiaodong Shi, "Deep semantic role labeling with self-attention," in Proc. AAAI, 2018.

[18] Xiaodong Zhang and Houfeng Wang, "A joint model of intent determination and slot filling for spoken language understanding.," in Proc. IJCAI, 2016, vol. 16, pp. 2993-2999.

[19] Dilek Hakkani-Tür, Gökhan Tür, Asli Celikyilmaz, Yun-Nung Chen, Jianfeng Gao, Li Deng, and Ye-Yi Wang, "Multi-domain joint semantic frame parsing using bi-directional rnn-lstm.," in Proc. Interspeech, 2016, pp. 715-719.

[20] Bing Liu and Ian Lane, "Attention-based recurrent neural network models for joint intent detection and slot filling," arXiv preprint arXiv:1609.01454, 2016.

[21] Changliang Li, Liang Li, and Ji Qi, "A self-attentive model with gate mechanism for spoken language understanding," in Proc. EMNLP, 2018, pp. 3824-3833.

[22] Yue Zhang and Jie Yang, "Chinese ner using lattice lstm,” in Proc. ACL, 2018, pp. 1554-1564.

[23] Tao Gui, Yicheng Zou, Qi Zhang, Minlong Peng, Jinlan $\mathrm{Fu}$, Zhongyu Wei, and Xuan-Jing Huang, "A lexiconbased graph neural network for chinese ner," in Proc. EMNLP, 2019, pp. 1039-1049.

[24] Xue Qiao, Chen Peng, Zhen Liu, and Yanfeng $\mathrm{Hu}$, "Word-character attention model for chinese text classification," International Journal of Machine Learning and Cybernetics, vol. 10, no. 12, pp. 3521-3537, 2019.

[25] Hanqing Tao, Shiwei Tong, Hongke Zhao, Tong Xu, Binbin Jin, and Qi Liu, "A radical-aware attention-based model for chinese text classification," in Proc. AAAI, 2019, vol. 33, pp. 5125-5132. 